\title{
Computational fluid dynamics analysis of a mixed flow pump impeller
}

\author{
A. Manivannan \\ Department of Mechanical Engineering, PSG College of Technology Coimbatore, INDIA \\ "Corresponding Author: e-mail: mani1496@yahoo.com, Tel +91-9486230632, Fax.+91-0422-2
}

\begin{abstract}
To improve the efficiency of mixed flow pump, Computational Fluid Dynamics (CFD) analysis is one of the advanced tools used in the pump industry. A detailed CFD analysis was done to predict the flow pattern inside the impeller which is an active pump component. From the results of CFD analysis, the velocity and pressure in the outlet of the impeller is predicted. CFD analyses are done using Star CCM+ software. These outlet flow conditions are used to calculate the efficiency of the impeller. The calculated value of efficiency from the empirical relations is 55\%. The optimum inlet and outlet vane angles are calculated for the existing impeller by using the empirical relations. The CAD models of the mixed flow impeller with optimum inlet and outlet angles are modeled using CAD modelling software ProE WF3. To find the relationship between the vane angles and the impeller performance the optimum vane angle is achieved step by step. Three CAD models are modeled with the vane angles between existing and optimum values. These models are analyzed individually to find the performance of the impeller. In the first case, outlet angle is increased by $5^{\circ}$. From the outlet flow conditions, obtained from the CFD analysis, it is evident that the reduced outlet recirculation and flow separation cause the improved efficiency. By changing the outlet angle the efficiency of the impeller is improved to $59 \%$. In the second case inlet angle is decreased by $10 \%$. The efficiency of the impeller in this case is $61 \%$. From this analysis it is understood that the changes in the inlet vane angle did not change the efficiency of the impeller as much as the changes in outlet angle. In the third case, impeller with optimum vane angles is analyzed and the outlet flow conditions are predicted. From the CFD analysis the efficiency of the impeller with optimum vane angles is calculated as $65 \%$. Thus, efficiency of the mixed flow impeller is improved by $18.18 \%$ by changing the inlet and outlet vane angles.
\end{abstract}

Keywords: Mixed flow pump, computational Fluid Dynamics (CFD) analysis, impeller design.

\section{Introduction}

A wide variety of centrifugal pump types have been constructed and used in many different applications in industry and other technical sectors. However, their design and performance prediction process is still a difficult task, mainly due to the great number of free geometric parameters, the effect of which cannot be directly evaluated. The significant cost and time of the trial-and-error process by constructing and testing physical prototypes reduces the profit margins of the pump manufacturers. For this reason CFD analysis is currently being used in the design and construction stage of various pump types.

The experimental way of pump test can give the actual value of head developed, power rating and efficiency. But the internal flow conditions cannot be predicted by the experimental results. From the CFD analysis software and advanced post processing tools the complex flow inside the impeller can be analyzed. The complex flow characteristics like inlet pre-swirl, flow separation and outlet recirculation cannot be visualized by the experimental way of pump test. But in the case of CFD analysis the above flow characters can be visualized clearly. Moreover design modification can be done easily and thus CFD analysis reduces the product development time and cost. Mixed flow pumps are widely used for water transportation or as cooling water pumps in power stations. Their operating range spans from full-load down to close to the shut-off head. In order to develop a reliable machine for this highly demanding operation, the behavior of the flow in the entire pump has to be predicted by a reliable computational method (Patel and Ramakrishnan, 2006).

The numerical simulation can provide quite accurate information on the fluid behaviour in the machine, and thus helps the engineer to obtain a thorough performance evaluation of a particular design. However, the challenge of improving the hydraulic efficiency requires the inverse design process, in which a significant number of alternative designs must be evaluated (Nagnostopoulos, 2006, Sedlar et al., 2009). The complex flow pattern inside the centrifugal pump is strong three dimensional with recirculation flows at inlet and exit, flow separation, cavitations. The curvatures of the blades and the rotational system have great influence on the flow field (Cheah and Lee, 2007). The meridional velocity and its effect on the performance of the pump can be improved by using proper vane geometric features (Miyauchi et al., 2004). Also the efficiency of the impeller can be improved by changing the volute design of the impeller and by increasing the number of impeller blades (Baun and Flack, 2003). 
In this work, a centrifugal pump impeller will be analyzed to predict the internal flow and the impeller performance. The results of the predicted flow field in the pump impeller and the effect of several design variables will be discussed. Steady state simulation using STAR CCM+ is used for the prediction of the flow in a single stage.

\section{Analysis of Mixed Flow Impeller}

In this work, the mixed flow pump detailed geometric feature of the impeller was studied and parameterization of impeller geometry was done. Parameterization was done by reducing number of controlling geometric variables, facilitating the investigation of their individual or combined effects on the flow and the impeller performance. The specification of the pump is given in the Table 1.

Table 1 Specification of the pump

\begin{tabular}{|c|c|}
\hline Bore size & $152.4 \mathrm{~mm}$ \\
\hline Capacity & $40 \mathrm{~m} 3 / \mathrm{hr}$ \\
\hline Number of stages & 6 \\
\hline Head & 28 m per stage \\
\hline Flow type & Mixed flow \\
\hline
\end{tabular}

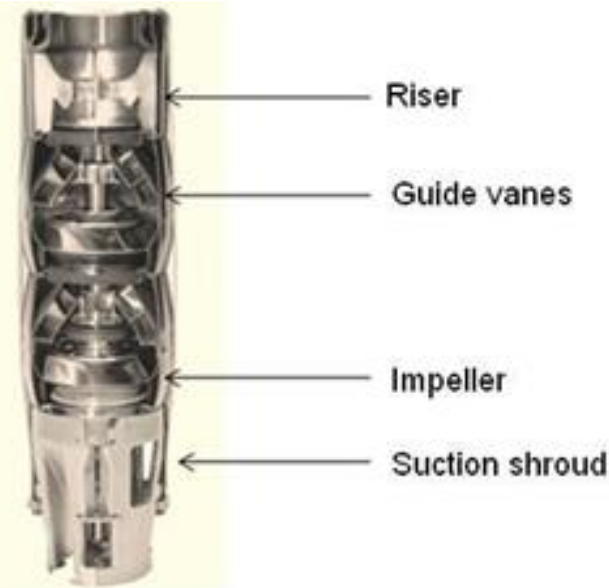

Figure 1. Mixed flow pump

A typical submersible pump consists of impeller, diffuser, riser, discharge adapter, intermediate bowl and shaft is shown in Fig.1. The performance of a pump depends on active pump components. The active pump components of a mixed flow pump are mixed flow impeller and guide vanes. These parts are important while analyzing a pump because mechanical energy (rotation) is transferred into fluid energy (pressure, velocity) by the active pump components.

The geometry of a mixed flow pump impeller is highly complex in nature. The mixed flow geometry consists of several features among those following geometric features are important as they have direct effect on overall pump performance (Patel and Ramakrishnan, 2006).

Table 2 Existing mixed flow impeller dimensions

\begin{tabular}{|l|l|}
\hline Parameter & Size \\
\hline Impeller inlet $(\mathrm{Di})$ & $75 \mathrm{~mm}$ \\
\hline Impeller outlet $(\mathrm{Do})$ & $105 \mathrm{~mm}$ \\
\hline Blades number & 6 \\
\hline Inlet angle $(\alpha)$ & 750 \\
\hline Outlet angle $(\beta)$ & 550 \\
\hline Blade thickness $(\mathrm{t})$ & $1.25 \mathrm{~mm}$ \\
\hline Blade inlet height $(\mathrm{Li})$ & $21 \mathrm{~mm}$ \\
\hline Blade inlet height $(\mathrm{Lo})$ & $16 \mathrm{~mm}$ \\
\hline
\end{tabular}

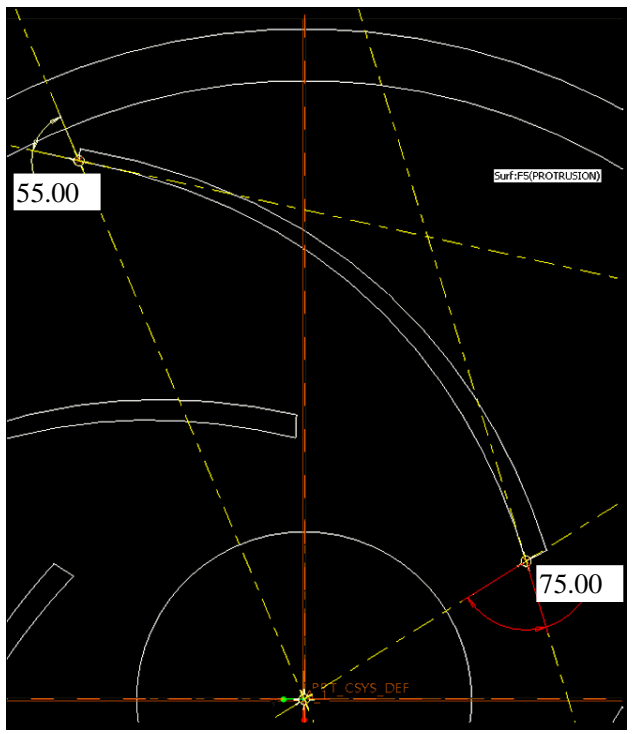

Figure 2. Existing Impeller vane dimensions

Geometric feature of the impeller was studied in detail and parameterization of impeller geometry was done. Parameterization was done by reducing number of controlling geometric variables (inlet angle, outlet angle), to investigate their individual or combined effects on the flow and the impeller performance (Patel and Ramakrishnan, 2006). After parameterization mixed flow 
impeller can be represented using a relatively small number of parameters; some of them are shown in Fig.2. and their values are given in Table 2. The rotation speed and the main impeller dimensions, namely the exit diameter and width $D 2$ and $b 2$, as well as the blade inlet and exit angles, $\beta 1$ and $\beta 2$, respectively, determine the nominal head and volume flow rate of the impeller.

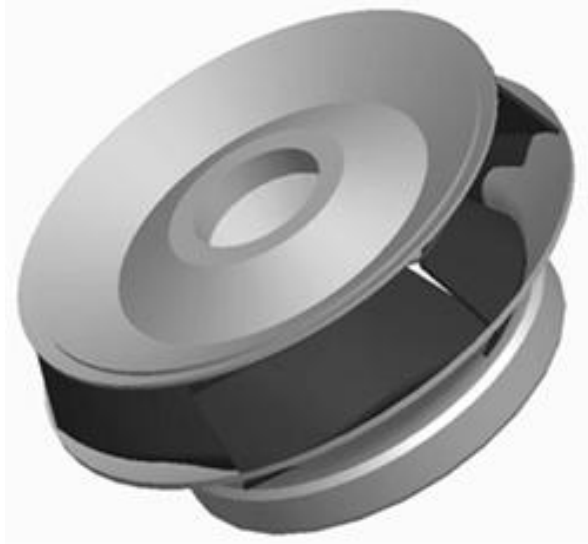

Figure 3. Model of mixed flow impeller

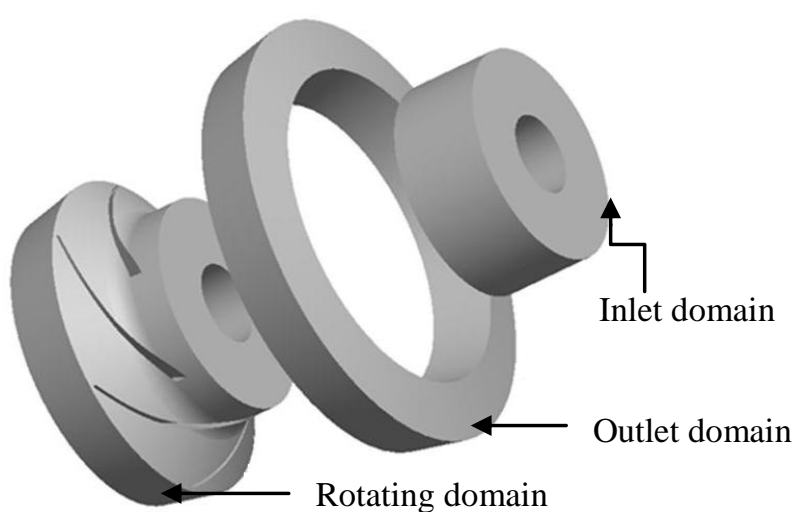

Figurer 4 Model of mixed flow impeller

The model of a mixed flow impeller is shown in Fig.3. The computational domain consists of the single passage of rotor, inlet domain and outlet domain. The corresponding model is shown in Fig.4. The final mesh of rotor and stator was generated using commercial software Star Design3.2. The total number of nodes was 81000 and 32000 for the rotor and the stator respectively. The current numerical computation is carried out with a multiple frame of reference approach because the impeller flow field is within a rotating frame whereby the intake section and outlet are in stationary frame. The meshes of three computational domains, the intake section, impeller and outlet, are generated separately.

Present numerical computation is based on steady-state condition with the following boundary conditions imposed: at the inlet of the computational domain the mass flow rate, the turbulence intensity, and a reference pressure are specified. The inlet flow velocity was specified as $5 \mathrm{~m} / \mathrm{s}$. Turbulent intensity was specified to be $5 \%$. At the outlet, pressure was specified as well. All the other variables are free to float. The volute casing and intake section walls are in stationary frame and modeled using a no-slip boundary condition. The modeled impeller was analyzed using the software Star CCM+. The boundary conditions and the best efficiency point for the mixed flow impeller are required for the CFD analysis of the impeller. For this hydraulic test was carried out for wide operating range. After conducting the detailed experimental study the hydraulic efficiency of mixed flow pump was calculated. Test results are used to obtain the boundary conditions and to find the best efficiency point for the mixed flow impeller.

\section{Design Modifications}

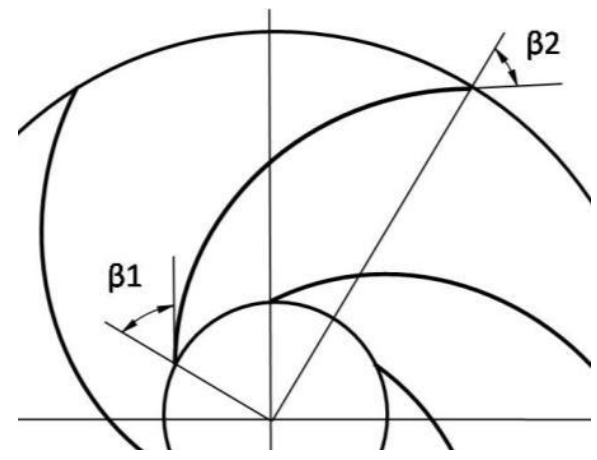

Figure 5. Vane angles

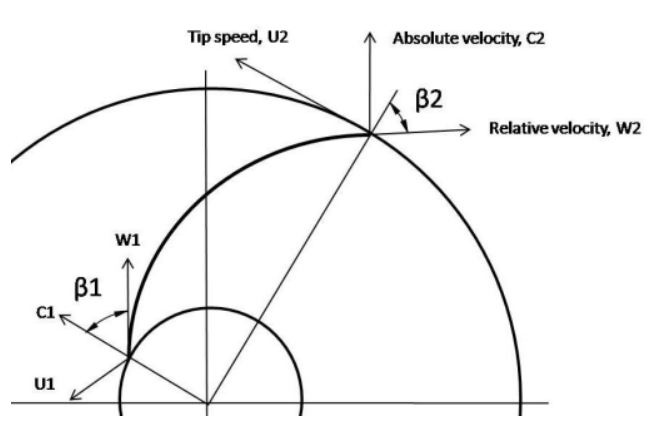

Figure 6 Flow pattern

The existing impeller is modified to get better results. The optimum value of impeller is calculated by using the empirical relations. The rotating impeller imparts energy to the fluid. It is the most important, the only rotating element in the pump. In mixed flow pumps and inlet of radial flow pumps, the streamlines are inclined from the axial direction toward the radial direction (Fig. 5). Impeller geometric features have great impact on the performance of the pump. The shape of the blade and the resulting flow pattern in the impeller determine how much energy is transferred by a given size impeller and how efficiently it operates (Fig. 6)

The performance of the pump is greatly depends on the impeller performance. Efficiency of the impeller depends on flow condition inside the impeller and geometric features of the impeller. The flow conditions inside the impeller can be varied by changing the geometric features of the impeller. Controllable geometric features of the impeller are given in Table 3. 
Table 4 Existing and optimum vane angles

Table 3 Geometric features of the impeller

\begin{tabular}{|l|l|l|}
\hline S. No & Parameter & Size \\
\hline 1 & Inlet diameter $(\mathrm{Di})$ & $75 \mathrm{~mm}$ \\
\hline 2 & Outlet diameter $(\mathrm{Do})$ & $105 \mathrm{~mm}$ \\
\hline 3 & Blades number & 6 \\
\hline 4 & Inlet angle $(\alpha)$ & $75^{\circ}$ \\
\hline 5 & Outlet angle $(\beta)$ & $55^{\circ}$ \\
\hline 6 & Blade thickness $(\mathrm{t})$ & $1.25 \mathrm{~mm}$ \\
\hline 7 & Blade inlet height $(\mathrm{Li})$ & $21 \mathrm{~mm}$ \\
\hline 8 & Blade inlet height $(\mathrm{Lo})$ & $16 \mathrm{~mm}$ \\
\hline
\end{tabular}

\begin{tabular}{|l|l|l|}
\hline Impeller design & $\begin{array}{l}\text { Inlet angle } \\
(\mathbf{d e g})\end{array}$ & $\begin{array}{l}\text { Outlet angle } \\
(\mathbf{d e g})\end{array}$ \\
\hline Existing & 75 & 55 \\
\hline Optimum & 55 & 65 \\
\hline
\end{tabular}

Table 5 Vane angles of modified impellers

\begin{tabular}{|c|c|c|}
\hline Impeller design & Inlet angle (deg) & Outlet angle (deg) \\
\hline Existing & 75 & 55 \\
\hline Impeller 1 & 75 & 60 \\
\hline Impeller 2 & 65 & 60 \\
\hline Impeller 3 & 55 & 65 \\
\hline
\end{tabular}

\subsection{Inlet Blade Angle Calculation}

Inlet angle of an impeller is calculated based on the inlet flow condition. The inlet diameter and the velocities at the inlet $\mathrm{C}_{1}$ and $\mathrm{U}_{1}$ determine the inlet blade angle $\beta_{1}$. The actual blade angle is given by the following relationship (Baun and Flack, 2003).

$$
\tan \beta_{1}=\frac{U_{1}}{C_{1}}
$$

The circumferential velocity $\left(\mathrm{U}_{1}\right)$ and axial velocity $\left(\mathrm{C}_{1}\right)$ depend on the inlet diameter, rotational speed and discharge. The circumferential velocity and axial velocity can be calculated from the following equations (Baun and Flack, 2003).

$$
\begin{aligned}
\mathrm{U}_{1} & =\frac{\omega \cdot D_{1}}{2} \\
\mathrm{C}_{1} & =\frac{4 Q}{\pi D^{2}}
\end{aligned}
$$

Based on the above equation impeller with optimum vane angles have to be modeled and analyzed to obtain the effect of vane angles on impeller performance. The optimum values of inlet angle and outlet angle were calculated. The optimum and current values of impeller angles are given in Table 4. The impeller inlet angle and outlet angle were changed step by step to know the relationship between these angles and impeller performance. Three impellers are modeled with various inlet and outlet angles. These impellers will be analyzed separately to find the efficiency. Table 5 shows the inlet and outlet angles of modified impellers.

The current impeller is modified based on the inlet and outlet angle calculation. These CAD models are analyzed to find the efficiency of the impeller. The inlet vane angle and outlet vane angle are changed step by step. The other impeller geometric features like blade thickness, shroud height and shroud diameter are kept as in case of existing impeller. These three impellers were modeled by changing the inlet and outlet vane angles of the existing impeller. Any changes made in a part will affect the whole assembly. This phenomenon is used to model the three impellers. The assembly of the impeller domain is copied to a new file. Rotor part is edited to change the inlet and outlet vane angles. This change is automatically taken place in the assembly also. Thus, the three impellers were modeled using the part relationship principle.

Separate computational grids were generated for the impeller and interface region. The type of mesh and mesh size was selected based on the research work done by Patel and Ramakrishnan (2006). The impeller was analyzed in the rotating frame of reference and the flow outside the impeller was analyzed in the stationary frame of reference. All simulations were carried out using water as the pumping liquid. At the inlet of the impeller the total pressure and the flow direction were specified whereas at the outlet the mass flow rate was fixed based on the condition to be investigated. The rotation rate for impeller is set to $2850 \mathrm{rpm}$ (Geis and Ebner, 2001). Solver should be selected in such a way that the type of analysis needed is validated properly by the solver. To analyze the mixed flow impeller the type of analysis used is moving frame of reference. This analysis type is valid one to analyze the mixed flow impeller as the impeller is the rotating part with rotation rate $2850 \mathrm{rpm}$. Each impeller is analyzed for different mass flow rate. For every analysis the reports were generated to obtain the area averaged values of pressure and velocity in the outlet of the impeller. These outlet flow conditions were used to calculate the head developed, power rating and efficiency of the impeller.

\section{Results and Discussion}

Fig.7 and Fig.8 show the resulting contours of velocity and pressure at a plane section that cross the impeller normal to- and through its axis of rotation 


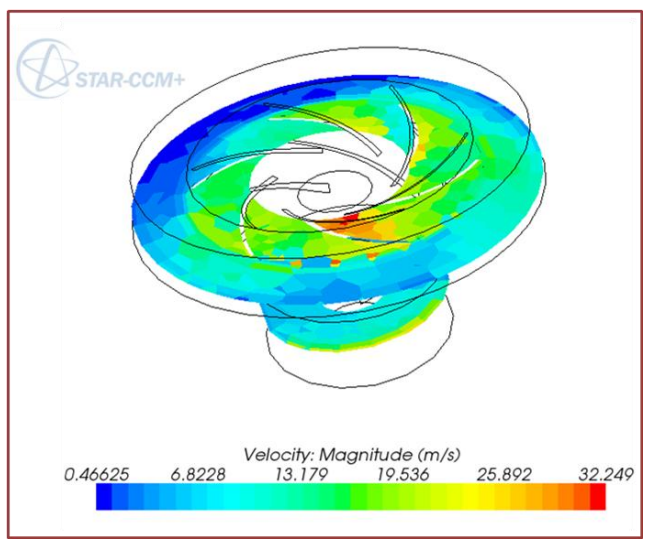

Figure 7. Velocity contours at $5 \mathrm{~m} / \mathrm{s}$ inlet velocity

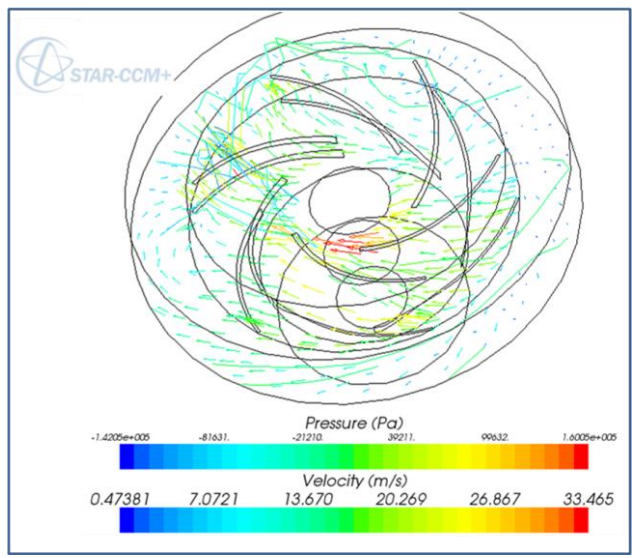

Figure 9. Isobar line

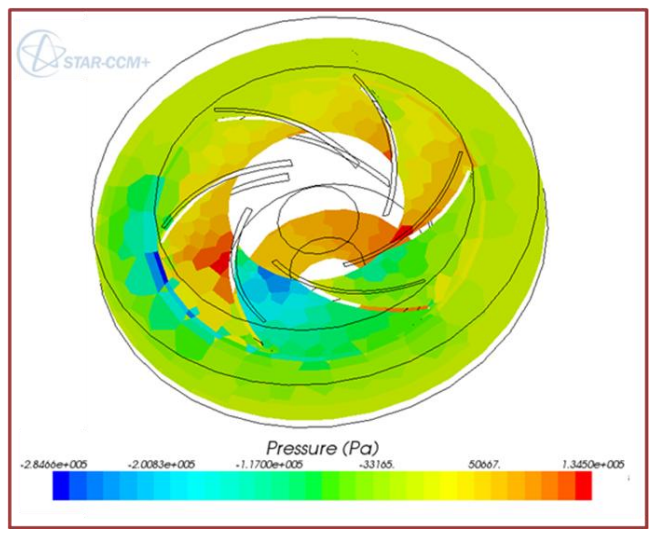

Figure 8. Velocity contours at $5 \mathrm{~m} / \mathrm{s}$ inlet velocity

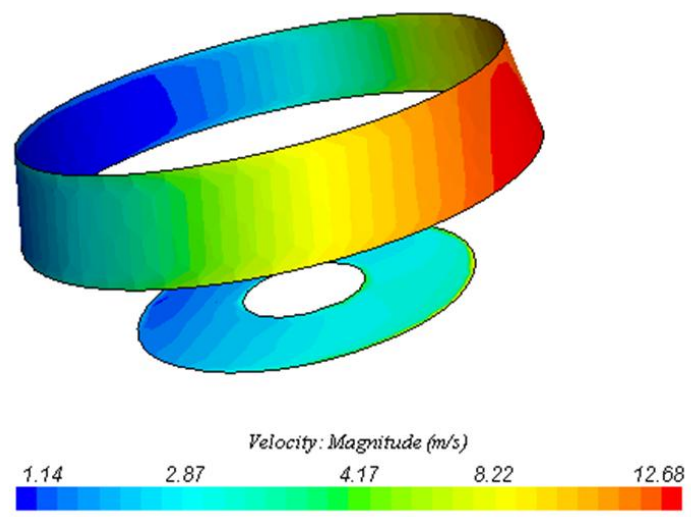

Figure 10. Averaged velocity distribution

As the flow entering the impeller eye, it is diverted into the blade-to-blade passage. Due to the unsteady effect developed at upstream, the flow entering the passage is no longer tangential to the leading edge of impeller blade. Separation of flow can be observed at all passages leading edge (Baun and Flack, 2003). Fig. 7 shows the velocity vector field with separation and inflow. Increased flow velocity can be observed at the blade inlet due to the blockage of the flow, whereas on the contrary the pressure is reduced (Fig.8). Further downstream the contours become smooth between the blades and the pressure increases continuously towards the exit of the computational domain. The isobar lines (Fig. 9) are not all perpendicular to the pressure side of the blade inside the impeller passage, this indicated that there could be a flow separation (Baun and Flack, 2003) because of the pressure gradient effect.

The hydraulic test was done on existing impeller and the results are compared with modified impeller CFD results. The k-e turbulence model can be used to analyze the mixed flow impeller and area averaged value of pressure and velocity can be used to calculate the head, efficiency and power rating of the impeller (Miner, 2001). To find the performance of modified impellers averaged velocities were taken (Fig.10) (Dupont, 2003; Miner, 2001).

\subsection{Head Variation}

The characteristic curve between discharge and head is given in Fig.11. It shows that for the existing impeller the head decreases while discharge increases. While discharge rate increases the velocity of fluid also increases and this increased velocity leads to pressure drop. Thus head reduces while discharge increases. The characteristic curve between discharge and head for the modified impeller1 shows that manometric head is increased by $3.22 \%$ at best efficiency point. The increased outlet angle leads to smooth flow in exit region. Thus the increase in outlet angle results in flow losses in outlet region. Inlet pre-swirl and flow separation are the two phenomena that occurred due to poor inlet design of impeller. By reducing the impeller inlet angle these losses can be minimized. The characteristic curve between discharge and head curve for the modified impeller2 shows that Head developed by IMP2 is $10.29 \%$ higher than the existing impeller. This higher head is developed because of reduced inlet pre-swirl and flow separation (Miyauchi et al., 2004). The manometric head developed by the impeller 3 is $13.66 \%$ higher than the existing 
impeller. The characteristic curve between discharge and head for the modified impeller3 shows that this impeller, with optimum inlet and outlet angles, has less deviation in manometric head for the complete operating range.

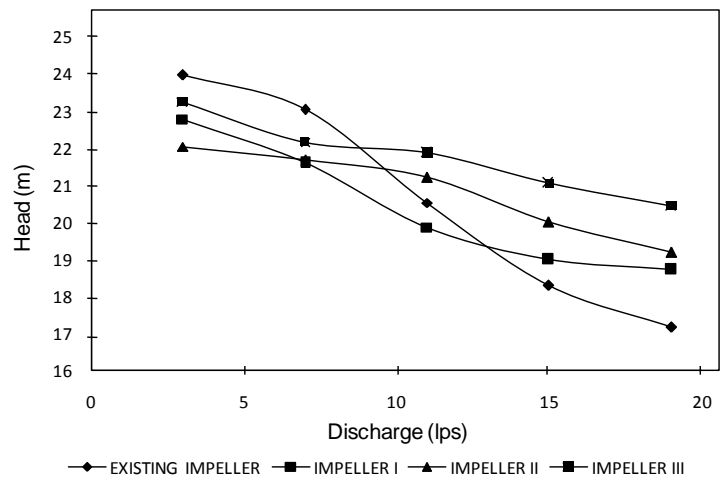

Figure 11. Head developed by the existing and modified impellers

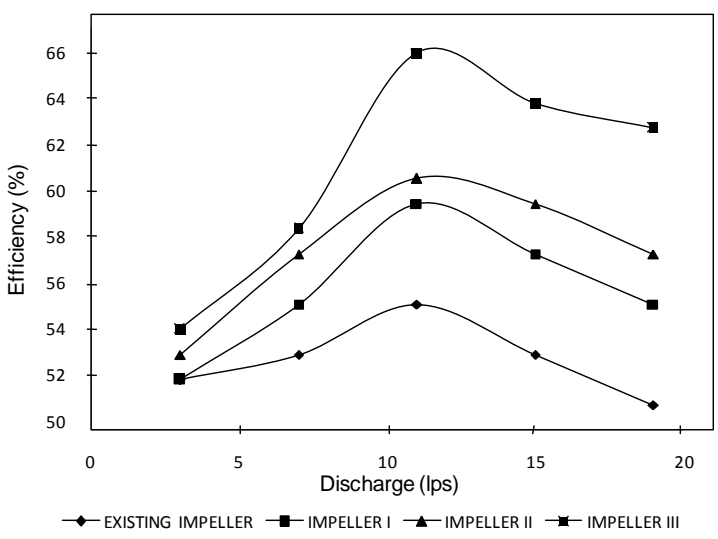

Figure 12. Efficiency of the existing and modified impellers

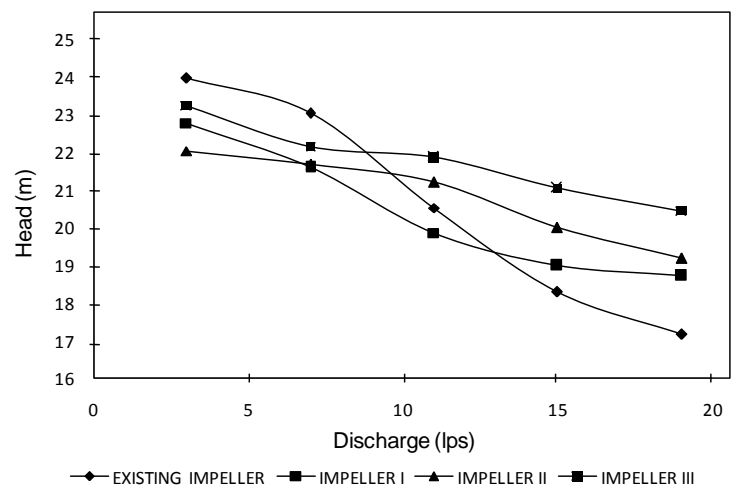

Figure 13. Power rating of the existing and modified impellers

\subsection{Efficiency Variation}

The characteristic curve between discharge and overall efficiency is given in Fig.12. It shows that overall efficiency for the existing impeller starts decreasing after $11 \mathrm{lps}$. Due to high discharge rate, velocity of the fluid increases and this high velocity cause cavitation losses and recirculation inside the impeller. Thus efficiency is decreasing while operating in high discharge rate. The characteristic curve between discharge and efficiency for impeller1 shows that efficiency of the impeller increases by $7.27 \%$. Outlet recirculation is an important phenomenon affecting the efficiency of the impeller (Baun and Flack, 2003). By increasing the value of outlet angle exit recirculation can be reduced. The characteristic curve between discharge and efficiency for impeller2 shows that efficiency of the impeller increases by $10.91 \%$. From the CFD results, it is evident that the changes in inlet angle did not make much deference in the hydraulic efficiency of the impeller when compared with changes in outlet angle. The characteristic curve between discharge and efficiency for impeller3 shows that efficiency of the impeller increases from by $18.18 \%$. The higher efficiency is achieved by selecting optimum values of inlet and outlet angles which otherwise lead to many flow losses discussed in previous chapters. Thus efficiency of the mixed flow impeller can be increased by $10 \%$ by changing inlet and outlet angle alone.

\subsection{Power Rating Variation}

The characteristic curve between discharge and power rating is given in Fig.13. It is understood from the graph that the power required to drive the pump increases from the existing impeller to the modified impellers. The increased blade loading during the operation may be the reason for the increase in power rating (Tuzson, 2000). The power rating for the modified impeller 1 is increased by $3.9 \%$. The power rating for the modified impeller 2 is increased by $7.61 \%$. The power rating for the modified impeller 3 is increased by $12.16 \%$. 


\section{Conclusions}

Based on the detailed design and CFD analysis of the mixed flow impeller, the following conclusions are derived.

- the mixed flow pump the best efficiency point of the pump is found to be $11 \mathrm{lps}$.

- the existing impeller, the head, power rating and efficiency are found out to be $19.24 \mathrm{~m}, 9.46 \mathrm{~kW}$ and $55 \%$ respectively.

- the impeller 1 , the percentage increase in the head, power rating and efficiency are $3.22 \%, 3.9 \%$ and $7.27 \%$ respectively.

- the impeller 2, the percentage increase in the head, power rating and efficiency are $10.29 \%, 7.61 \%$ and $10.91 \%$ respectively.

- the impeller 3, the percentage increase in the head, power rating and efficiency are $13.66 \%, 12.16 \%$ and $18.18 \%$ respectively. Based on the above it is concluded that impeller 3 gives better performance. Thus CFD analysis is an effective tool to calculate quickly and inexpensively the effect of design and operating parameter of pump. By properly designing pump impeller the efficiency of pump can be improved.

\section{Nomenclature \\ $\mathrm{C}_{1}$ - axial velocity $(\mathrm{m} / \mathrm{s})$ \\ $\mathrm{D}_{1}$ - inlet diameter $(\mathrm{m})$ \\ $\mathrm{Q}$ - discharge $\left(\mathrm{m}^{3} / \mathrm{s}\right)$ \\ $\mathrm{U}_{1}$ - circumferential velocity $(\mathrm{m} / \mathrm{s})$ \\ $\beta_{1}$ - inlet blade angle (Deg) \\ $\omega$ - angular velocity $(\mathrm{deg} / \mathrm{s})$}

\section{Acknowledgement}

The Author would like to thank S. Suthan, R. Godwin Bennet and J. Madhanraj, Mechanical Engg. students for their contributions in this paper.

\section{References}

Baun D.O., Flack R.D. 2003. Effects of volute design and number of impeller blades on lateral impeller forces and hydraulic performance, International Journal of Rotating Machinery, Vol. 2, No. 9, pp. 145-152.

Cheah K.W., Lee T. S. 2007, Numerical flow simulation in a centrifugal pump at design and off-design conditions, International Journal of Rotating Machinery, Vol. 2007, Article ID 83641, doi: 10.1115/2007/83641, 8 p.

Dupont P. 2003, Cavitating flow calculations in industry, International Journal of Rotating Machinery. Vol. 9, No. 3, pp. 171-179.

Geis T. and Ebner J. 2001, Flow structures inside a rotor-stator cavity, International Journal of Rotating Machinery, Vol. 7, No. 4, pp. 285-300.

Miner S.M. 2001, 3-D viscous flow analysis of a mixed flow pump impeller, International Journal of Rotating Machinery, Vol. 7, No. 1, pp. 53-63.

Miyauchi S., Horiguchi H. and Fukutomi J.-I., Takahashi A., 2004, Optimization of meridional flow channel design of pump impeller, International Journal of Rotating Machinery, Vol. 10, pp. 115-119.

Nagnostopoulos J. 2006. CFD analysis and design effects in a radial pump impeller, WSEAS Transactions on Fluid Mechanics, Vol.1, pp. 763-769.

Patel K., Ramakrishnan N. 2006, CFD analysis of mixed flow pump, International ANSYS Conference Proceedings.

Sedlar M., Soukal J. and Komarek M. 2009. CFD analysis of middle stage of multistage pump operating in turbine regime, Engineering Mechanics, Vol.16, No.6, p.413-421.

Tuzson J. 2000, Centrifugal pump design, Wiley Inter-Science Publications, New York, USA, 2000.

Biographical notes

Manivannan. A completed BE in Mechanical Engineering from RVS College of Engg. and Technology, Dindigul and received M.E and Ph.D. from Anna University, Chennai, India in 2001 and 2008, respectively. He is a faculty in the Department of Mechanical Engineering, PSG College of Technology, Coimbatore, India. He has more than 10 years of experience in teaching and research. His current area of research includes internal combustion engines, energy engineering and CFD. He is currently dealing with few projects for various sponsoring agency.

Received July 2010

Accepted November 2010

Final acceptance in revised form November 2010 